\title{
Only patients with dysplasia progress to adenocarcinoma in Barrett's oesophagus
}

\author{
M Miros, P Kerlin, N Walker
}

\begin{abstract}
Columnar lined oesophagus (Barrett's oesophagus) carries a risk for the development of adenocarcinoma. Epithelial dysplasia appears to be a precursor but the utility of this marker for predicting subsequent adenocarcinoma is unsettled. We therefore prospectively studied 81 patients with histologically proven columnar epithelium of at least the distal $3 \mathrm{~cm}$ of the tubular oesophagus with regular endoscopic biopsies for a total of 289.2 patient years (mean 3.6 years, range $0.5-8$ ). Twenty three patients (28\%) had epithelial dysplasia detected during follow up. Both patients with persistent high grade dysplasia present on initial biopsies developed adenocarcinoma after 2.6-4.5 years, despite the absence of gross macroscopic change. The initial single layer pleomorphic high grade dysplasia in one patient regressed to low grade dysplasia which has persisted for 1.5 years. Of 10 patients with initial low grade dysplasia, one progressed to adenocarcinoma in 4.3 years. The low grade dysplasia persisted unchanged in seven patients for 1.5-7 years and appears to have regressed in two patients after three to five years. Ten patients developed low grade dysplasia during the surveillance period. This has persisted unchanged in six patients from $0.5-5$ years, regressed in three for $0.5-5$ years and has appeared after the first yearly biopsy in one patient. No patient without dysplasia has developed adenocarcinoma. The incidence of adenocarcinoma in Barrett's oesophagus in this study is one case per 96 patient years. This is 61 times $(95 \%$ confidence limits $12-176)$ the age adjusted incidence of oesophageal cancer in Australia. Persistent high grade dysplasia appears to be a sensitive indicator for the development of subsequent adenocarcinoma.
\end{abstract}

Metaplastic columnar mucosa in the distal oesophagus, Barrett's oesophagus, carries an increased risk for the development of adenocarcinoma, ${ }^{1-3}$ of between one case per 46 to 441 patient years, depending on the type of follow up and selection bias. ${ }^{45}$ In view of this, patients with Barrett's oesophagus are usually entered into an endoscopic surveillance programme to detect epithelial dysplasia, although the rate of progression of dysplasia to adenocarcinoma is currently unknown..$^{6-8}$

The aims of this study were to determine prospectively the incidence of dysplasia and adenocarcinoma in unselected patients with Barrett's oesophagus and to determine the utility of dysplasia in predicting the development of future malignancy.
Methods

PATIENTS

All patients undergoing an upper gastrointestinal endoscopy at the Princess Alexandra Hospital, Brisbane, between October 1981 and October 1988 were assessed for the presence of Barrett's oesophagus. ' This was diagnosed if histologically proven columnar epithelium was present circumferentially in at least the distal $3 \mathrm{~cm}$ of the tubular oesophagus. To ensure that endoscopic pinch biopsy specimens containing columnar epithelium were from the oesophagus, details of the location of the squamocolumnar junction, the upper and lower limits of an hiatus hernia (if present) and the precise location of multiple biopsies were recorded. A mean of 19 patients (4.4) (range 13-25) were diagnosed with Barrett's oesophagus every year.

All Barrett's oesophagus patients without a concurrent adenocarcinoma on the initial endoscopy were then encouraged to enter a surveillance programme. Patients who agreed were reendoscoped at 12 monthly intervals, earlier if a Barrett's ulcer was present or dysplasia was detected. Patients with high grade dysplasia were reendoscoped immediately with multiple biopsies every $2 \mathrm{~cm}$ of the Barrett's oesophagus to eliminate a concurrent adenocarcinoma. If this was negative, they were rebiopsied at three monthly intervals. Patients with low grade dysplasia were followed at six monthly intervals. At each endoscopic visit, the length of the Barrett's oesophagus and the presence of macroscopic lesions (oesophagitis, stricture, ulcer, tumour) were recorded. Random four quadrant biopsies were taken of the columnar epithelium and any lesions present. Patients who missed their annual endoscopy appointment were contacted by telephone to arrange a new appointment. Patients refusing further endoscopies were assessed clinically for any oesophageal symptoms although they were considered to be lost to follow up in this study.

Specimens for histology were fixed in $10 \%$ formalin, embedded in paraffin wax and sections cut at $5 \mu \mathrm{m}$ and stained with haematoxylin and eosin ( $\mathrm{H} \& \mathrm{E})$. Slides were reviewed by one pathologist. Barrett's epithelium was classified using the histological criteria of Paull et $a l^{10}$ into gastric fundic type with parietal and chief cells, junctional type with cardiac mucous glands and specialised columnar epithelium with villiform surface, mucous glands and intestinal goblet cells (Fig 1). The presence of ulceration was recorded. Foveolar hyperplasia (elongation, tortuosity and hypercellularity of superficial pits lined by epithelium of gastric surface type) was differentiated from dysplasia. The latter was 


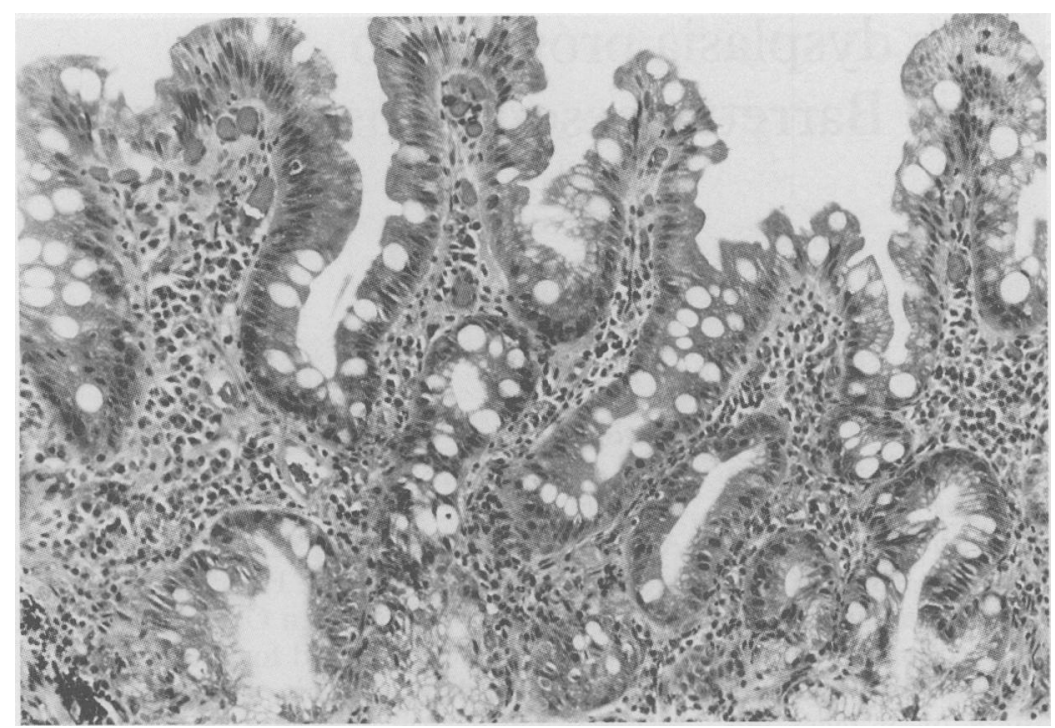

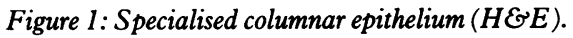

assessed using criteria established for inflammatory bowel disease. " Biopsies were classified as negative for dysplasia, indefinite for dysplasia or positive for dysplasia. The latter were graded as low or high grade dysplasia. As low grade dysplasia and changes indefinite for dysplasia cannot be reliably distinguished, ${ }^{12}$ these were placed in a single category. Dysplasia was recognised by the presence of cytological and architectural abnormalities in glandular epithelium. In low grade dysplasia, enlarged, crowded and stratified hyperchromatic nuclei were located predominantly in the cell base (Fig 2). In high grade dysplasia, abnormal nuclei regularly

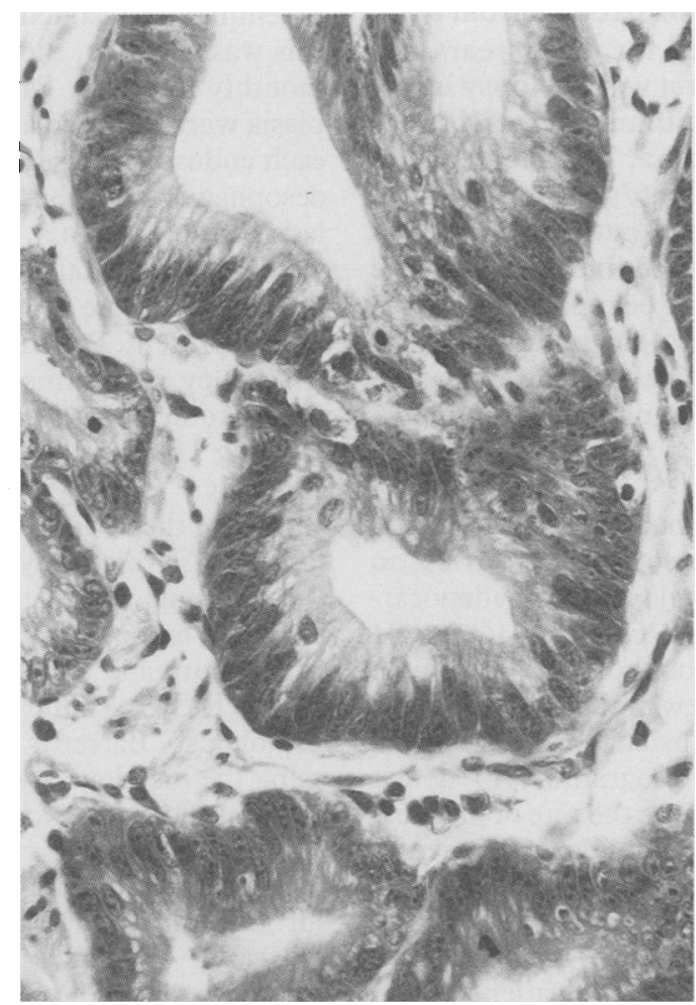

Figure 2: Low grade dysplasia of glandular epithelium. Compare with normal glandular epithelium extending to the middle left-hand margin $(H \mathcal{E} E)$.

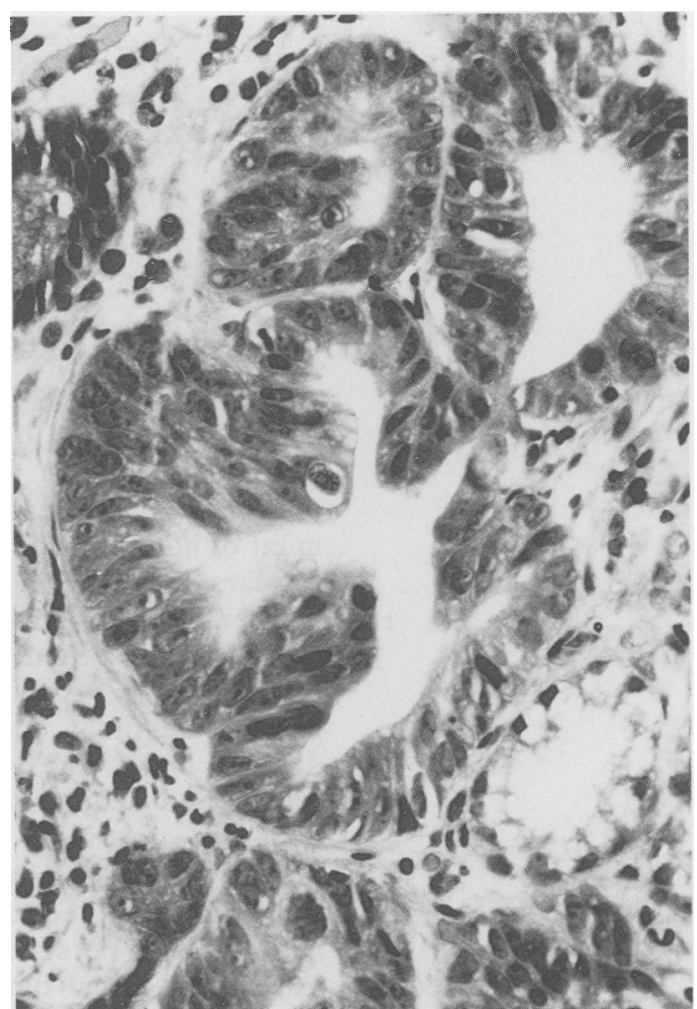

Figure 3: High grade dysplasia of glandular epithelium

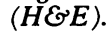

reached the cell apex (Fig 3$)^{213-15}$ and architectural changes approached those seen in adenocarcinoma. Type II epithelial dysplasia ${ }^{13}$ is was less frequently seen. It was characterised by enlarged rounded or pleomorphic vesicular nuclei with a peripheral rim of condensed chromatin and prominent nucleoli. Nuclear crowding and stratification did not occur.

Patients with reflux oesophagitis or a Barrett's ulcer were treated with $\mathrm{H}_{2}$ receptor blockers. A number of patients with resistant Barrett's ulcers or severe unresponsive oesophagitis were treated with omeprazole. Patients with strictures were treated with bougienage as necessary.

The prevalence of oesophageal cancer was calculated as the frequency of Barrett's oesophagus patients with adenocarcinoma at the initial endoscopy. The years of follow up in each patient were calculated as the interval between the first biopsy showing Barrett's oesophagus and the last follow up biopsy. The incidence was calculated from the number of patients developing adenocarcinoma during follow up and the total follow up in years. This incidence was compared with the annual age adjusted rate of oesophageal cancer in the Australian population $^{16}$ to give a standardised morbidity ratio. Ninety five per cent confidence limits were calculated using an exact Poisson distribution. ${ }^{17}$ Results are expressed as mean standard deviation (SD).

\section{Results}

One hundred and thirty three patients (male 101, female 32) were diagnosed as having Barrett's oesophagus over the seven year period. Adenocarcinoma of the oesophagus was found con- 
TABLE I Findings at initial endoscopy in 124 patients with Barrett's oesophagus

\begin{tabular}{lcr}
\hline & & $(n)$ \\
\hline Length of Barrett's segment (cm) & $3-4 \cdot 9$ & 57 \\
& $5-9 \cdot 9$ & 44 \\
& $>10$ & 23 \\
Associated mucosal lesions & Oesophagitis & 83 \\
& Stricture & 44 \\
Type of epithelium & Barrett's ulcer & 17 \\
& Specialised columnar & 110 \\
& Junctional & 116 \\
& Fundic & 32 \\
\hline
\end{tabular}

TABLE II Histologic changes during surveillance endoscopy in 13 patients with Barrett's oesophagus and dysplasia on initial biopsy

\begin{tabular}{llll}
\hline Patient & $\begin{array}{l}\text { Entrance } \\
\text { dysplasia }\end{array}$ & $\begin{array}{l}\text { Final } \\
\text { dysplasia }\end{array}$ & $\begin{array}{l}\text { Follow up } \\
(y r)\end{array}$ \\
\hline 1 HM & LGD & Negative & 3 \\
2 LW & LGD & Negative & 5 \\
3 NJ & LGD & LGD & $1 \cdot 5$ \\
4 TE & LGD & LGD & $2 \cdot 5$ \\
5 AM & LGD & LGD & 3 \\
6 AB & LGD & LGD & 3 \\
7 PL & LGD & LGD & $3 \cdot 5$ \\
8 JP & LGD & LGD & 4 \\
9 OK & LGD & LGD & 7 \\
10 CI & LGD & Adeno Ca & $4 \cdot 3$ \\
11 GW & HGD & Adeno Ca & $2 \cdot 6$ \\
12 JK & HGD & Adeno Ca & $4 \cdot 5$ \\
13 RC & HGD & LGD & 1.5 \\
\hline
\end{tabular}

LGD=low grade dysplasia; HGD= high grade dysplasia; Adeno $\mathrm{Ca}=$ invasive adenocarcinoma.

^Type II single layer pleomorphic dysplasia.

TABLE III Histologic changes in 10 patients with Barrett's oesophagus in whom dysplasia developed during the surveillance programme

\begin{tabular}{|c|c|c|c|c|}
\hline Patient & $\begin{array}{l}\text { Most severe } \\
\text { dysplasia } \\
\text { during the } \\
\text { surveillance }\end{array}$ & $\begin{array}{l}\text { Final } \\
\text { dysplasia }\end{array}$ & $\begin{array}{l}\text { Dysplasia } \\
\text { duration } \\
(y r)\end{array}$ & $\begin{array}{l}\text { Total } \\
\text { histologic } \\
\text { follow up } \\
(y r)\end{array}$ \\
\hline $\begin{array}{l}1 \mathrm{MB} \\
2 \mathrm{AM} \\
3 \mathrm{NH} \\
4 \mathrm{LB} \\
5 \mathrm{AP} \\
6 \mathrm{EP} \\
7 \mathrm{MG} \\
8 \mathrm{DP} \\
9 \mathrm{VT} \\
10 \mathrm{WS}\end{array}$ & $\begin{array}{l}\text { LGD } \\
\text { LGD } \\
\text { LGD } \\
\text { LGD } \\
\text { LGD } \\
\text { LGD } \\
\text { LGD } \\
\text { LGD } \\
\text { LGD } \\
\text { LGD }\end{array}$ & $\begin{array}{l}\text { Negative } \\
\text { Negative } \\
\text { LGD } \\
\text { Negative } \\
\text { LGD } \\
\text { LGD } \\
\text { LGD } \\
\text { LGD } \\
\text { LGD } \\
\text { LGD }\end{array}$ & $\begin{array}{l}0 \\
0 \\
0 \\
0 \cdot 5 \\
0 \cdot 5 \\
1 \\
1 \\
1 \\
1 \\
5 \cdot 5\end{array}$ & $\begin{array}{l}2 \\
5 \\
1 \\
5 \\
2 \cdot 5 \\
3 \\
5 \\
5 \\
5 \\
6\end{array}$ \\
\hline
\end{tabular}

LGD = low grade dysplasia

currently on initial biopsy in nine patients (prevalence 6.9\%). One of these patients had an initial biopsy showing only high grade dysplasia with repeat biopsies showing the invasive carcinoma. Eight of the nine patients had surrounding high grade dysplasia in their biopsies. The adjacent Barrett's mucosa contained gastric junctional type epithelium in all patients and specialised columnar epithelium in eight; none contained gastric fundic type epithelium. Six of these patients were treated by an Ivor Lewis oesophagectomy, one received chemotherapy and radiotherapy and two patients received no treatment. Two patients are currently alive 12 to 24 months after resection, the other patients dying two to 24 months after diagnosis of tumour.

The remaining 124 patients (male 93 , female 31 ) with a mean age of 63.3 years ( 14.9 years) were assessed for the surveillance programme: Initial endoscopic features of this group are listed in Table I.

Eighty one patients are currently entered into the surveillance programme with histological follow up for a total of $289 \cdot 2$ patient years (mean $3.6(1.7)$ years/patient, range $0.5-8.0$ years). The remaining 43 patients were lost to histological follow up. Twelve of these patients died from unrelated causes (cardiorespiratory seven, breast cancer two, lung cancer three) before the first year follow up biopsy. The remaining 31 patients (mean age 72 (18) years) refused further endoscopic follow up, predominantly because of other medical problems. Clinical follow up is available in 29 of these, for a total of 61 patient years (mean 2.0 years/patient). Five of the 29 have died of unrelated causes, while 24 have currently no oesophageal symptoms. Two patients have been lost completely to follow up.

Three patients have developed adenocarcinoma $2 \cdot 6-4 \cdot 5$ years after entering the surveillance programme. This is an incidence of adenocarcinoma in Barrett's oesophagus of one case per 96.4 patient years, or an annual incidence of 1037 cases per 100000 Barrett's oesophagus patients. Compared with the annual incidence of oesophageal cancer in the Australian community, the standardised morbidity ratio is $60 \cdot 5$ (95\% confidence limits, $12 \cdot 5-173 \cdot 8)$.

All three patients with adenocarcinoma were male (62, 65, 74 years) with a Barrett's length of at least $7 \mathrm{~cm}$, a history of previous severe reflux oesophagitis and multiple medical problems (respiratory disease three, ischaemic heart disease two, alcohol one). Two patients required maintenance omeprazole treatment for severe reflux oesophagitis or Barrett's ulceration. One of these patients had a Nissen's fundoplication carried out three years before the diagnosis of adenocarcinoma. In both these patients, the diagnosis of early invasive adenocarcinoma was made only on biopsy with no macroscopic lesion visible at endoscopy. One of these patients was treated with a radical oesophagectomy and tubularisation of the stomach. Histology revealed a moderately differentiated adenocarcinoma without invasion of the muscularis propria or lymph node metastases. Unfortunately, the patient died two weeks postoperatively with a myocardial infarct. The other patient with chronic obstructive airways disease was considered unfit for surgery. He remains asymptomatic 18 months after detection of cancer with only a small tumour nodule at endoscopy. The third patient, an alcoholic, initially refused to enter the surveillance programme but represented with progressive dysphagia $4 \cdot 3$ yrs later. Endoscopy revealed a large fungating poorly differentiated adenocarcinoma. Palliation was undertaken with an oesophageal bypass procedure but the patient died one week postoperatively of pulmonary thromboembolism.

Dysplasia was present on initial endoscopy in 13 patients (16\%) (Table II), three with high grade dysplasia and 10 with low grade dysplasia. Ten patients (12\%) subsequently developed low grade dysplasia during the surveillance programme (Table III). At the end of the surveillance programme, 18 patients $(22 \%)$ had dysplasia or adenocarcinoma. Barrett's mucosa in all patients with dysplasia contained gastric junctional type and specialised columnar 

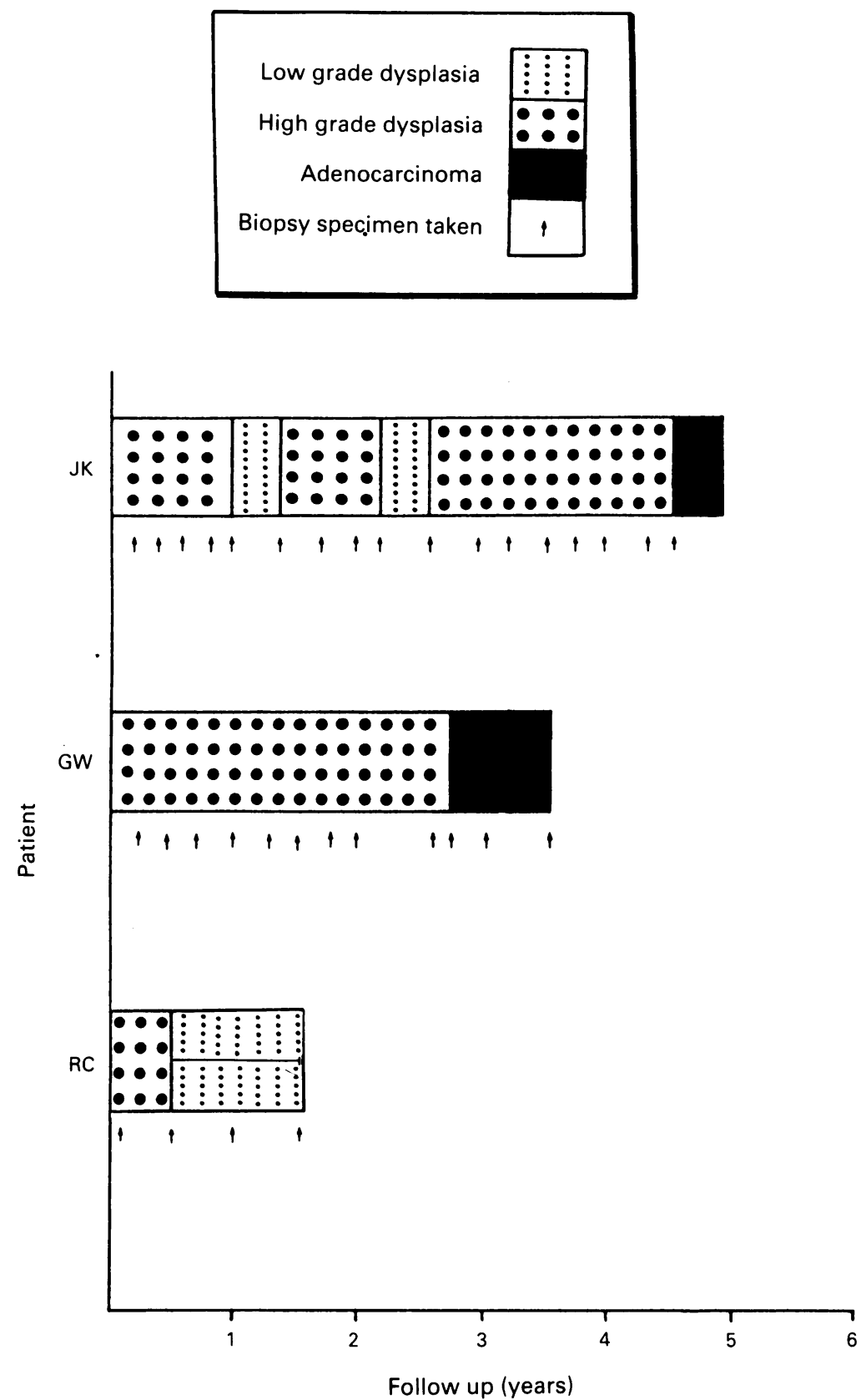

Figure 4: Histologic changes during surveillance endoscopy in three patients with Barrett's oesophagus and high grade dysplasia. Arrows indicate the timing of endoscopic biopsies.

epithelium; gastric fundic type epithelium was present in six patients with low grade dysplasia and one with high grade dysplasia. Preceding dysplasia was documented in all three patients who developed adenocarcinoma. High grade dysplasia persisted for $2 \cdot 6-4 \cdot 5$ years before the development of adenocarcinoma in two patients (Fig 4). The third patient had documented low grade dysplasia $4 \cdot 3$ years before the development of adenocarcinoma.

Unfortunately, intervening biopsies were unable to be carried out in this patient to assess any progression of the dysplasia before the development of adenocarcinoma. One other patient had an initial single biopsy showing type II high grade dysplasia at the time of severe oesophagitis. Multiple repeat biopsies after treatment of oesophagitis over the next 1.5 years, however, revealed only persistent low grade dysplasia. No other patient with low grade dysplasia has currently progressed to either high grade dysplasia or adenocarcinoma, despite its persistence, in 15 patients from $0 \cdot 5-7$ years. The low grade dysplasia appears to have regressed in three patients.

During the surveillance period, 21 of the 124 patients died (adenocarcinoma of oesophagus two, other malignancies nine, cardiorespiratory 10). A further five patients developed other malignancies (colon one, haemopoietic one, melanoma one, lung two).

\section{Discussion}

Patients with Barrett's oesophagus have an increased risk for the development of adenocarcinoma of the oesophagus. The reported incidence in retrospective studies has varied from one case per 170 to 441 patient years in postal studies $^{51819}$ to one case per 175 in an endoscopic study. ${ }^{21}$ As many patients may be asymptomatic for a number of years with early adenocarcinoma of the oesophagus, postal studies and endoscopic studies without biopsies underestimate the true incidence of adenocarcinoma. Two recently published series with prospective endoscopic biopsies have attempted to define the true annual incidence. Robertson et $a l^{21}$ diagnosed 56 patients with Barrett's oesophagus over a 10 year period. On follow up they found an incidence of one case per 56 patient years. Hameeteman et $a l^{22}$ followed 50 patients with Barrett's oesophagus diagnosed over a 14 year period to find an incidence of one case per 52 patient years. This compares with our incidence of one case per 96 patient years in 81 patients. If we include the 29 patients with clinical follow up only for a total of 61 patient years, then the lowest incidence in our study was one case per 117 patient years.

All recent incidence figures support the establishment of a surveillance programme to detect early carcinoma. Our incidence figures suggest, however, that 100 patients with Barrett's oesophagus need to be endoscoped every year to detect one case of adenocarcinoma. Other markers of increased risk are required to help target the population at greatest risk of developing malignancy. One possible marker is epithelial dysplasia. A recent consensus study for dysplasia ${ }^{13}$ found interobserver variation in high grade dysplasia is low with a reproducibility rate of $87 \%$. In low grade dysplasia, the reproducibility rate was lower at $70 \% .^{12}$ Despite this, dysplasia appears to be reliably associated with adenocarcinoma. Our prevalence data and several other studies, ${ }^{36}{ }^{15}$ found that dysplasia is usually present in epithelium adjacent to an adenocarcinoma. Although previous work has suggested a possible progression from low grade dysplasia to high grade dysplasia to invasive cancer, ${ }^{63}$ few longitudinal prospective studies are available.

Our results indicate that $28 \%$ of patients (at initial diagnosis $12 \%$, on follow up $16 \%$ ) with Barrett's oesophagus had dysplasia documented at least once during a follow up period. The majority $(90 \%)$ of these have had low grade dysplasia. This compares with previous incid- 
ence reports of dysplasia of $14 \%^{21}$ and $36 \%^{22}$ and prevalence rates of from $10 \%$ to $18 \% .2425$

The management of high grade dysplasia once detected is still controversial. Previous studies finding a high incidence of concurrent early adenocarcinoma in patients with high grade dysplasia ${ }^{6} 15$ led many researchers to recommend immediate oesophagectomy. Reid et $a l^{23}$ more recently showed that if multiple large biopsies are taken of areas with high grade dysplasia, then superimposed adenocarcinoma can be reliably detected before oesophagectomy. Few studies have prospectively followed patients with high grade dysplasia. Our study supports the conclusion that all patients with persistent high grade dysplasia will eventually develop adenocarcinoma although this may be up to 4.5 years later. Others have followed patients with high grade dysplasia for up to six years with no progression to adenocarcinoma. ${ }^{62326}$ Hameeteman et $a l^{22}$ prospectively followed seven patients with high grade dysplasia. Five progressed rapidly to adenocarcinoma. No progression to adenocarcinoma occurred in the other two patients over 36 and 44 months.

Little information is available about the development and progression of low grade dysplasia. Our results indicate that $20 \%$ of patients with Barrett's oesophagus will have documented low grade dysplasia during follow up. In only one patient did this dysplasia progress to an adenocarcinoma over a 4.3 year period. Unfortunately, no biopsies were taken in the intervening period to establish whether the low grade dysplasia progressed to high grade dysplasia before the development of adenocarcinoma. As this patient had low grade dysplasia on his initial biopsy, the total period of low grade dysplasia before progression to adenocarcinoma is uncertain. A further 18 patients with low grade dysplasia followed up for 0.5-7 years have not progressed. In contrast, five of 17 patients with low grade dysplasia developed high grade dysplasia and then adenocarcinoma over a $1 \cdot 5-6$ year period in the study by Hameeteman et al. ${ }^{22}$ Other modalities currently being investigated to determine more accurately which patients with low grade dysplasia develop adenocarcinoma include DNA flow cytometry ${ }^{27}$ and DNA image cytometry. ${ }^{29}$

It is our current policy to offer endoscopic surveillance to all patients with Barrett's oesophagus who are suitable and willing to have an oesophagectomy if adenocarcinoma is found. As patients without dysplasia do not progress to malignancy in the medium term, we recommend second yearly endoscopic biopsies for these patients. Patients with high grade dysplasia should have the dysplasia confirmed and adenocarcinoma excluded with immediate repeat endoscopic biopsies. If high grade dysplasia only is still present and the patient is young and fit, an oesophagectomy should be performed, as all patients eventually develop invasive adenocarcinoma. Older patients or patients with an increased operative risk should be carefully followed up every six months for the development of invasive cancer. If only low grade dysplasia is confirmed on two separate occasions, only yearly biopsies need to be done as the rate of progression to malignancy is slow. These recommendations would decrease the number of biopsies carried out for surveillance of Barrett's oesophagus whilst targeting patients with a higher risk of malignant transformation for more intensive efforts to diagnose early potentially curable adenocarcinoma.

A portion of this study was presented at the Annual Scientific Meeting of the Gastroenterological Society of Australia in Perth, April 1989.

1 Naef AP, Savary M, Ozzello L. Columnar lined lower esophagus: an acquired lesion with malignant predisposition. 7 Thorac Cardiovasc Surg 1975; 70: 826-35.

2 Skinner DB, Walther BC, Riddell RH, et al. Barrett's esophagus. Comparison of benign and malignant cases. Ann Surg 1983; 198: 554-66.

3 Haggitt RC, Tryzelaar J, Ellis FH, Colcher H. Adenocarcinoma complicating columnar epithelium-lined (Barrett's) esophagus. Am f Clin Pathol 1978; 70: 1-5.

4 Sprung DJ, Ellis FH, Gibb SP. Regression of Barrett's epithelium after anti-reflux surgery. [Abstract]. $A m \mathcal{F}$ Gastroenterol 1984; 79: 817.

5 Cameron AJ, Ott BJ, Payne WS. The incidence of adenocarcinoma in columnar-lined (Barrett's) esophagus. $N$ Engl F Med 1985; 313: 857-9.

6 Hamilton SR, Smith RRL. The relationship between columnar epithelial dysplasia and invasive adenocarcinoma arising in Barrett's esophagus. Am $\mathcal{F}$ Clin Pathol 1987; 87: 301-12.

7 Reid BJ, Weinstein WM. Barrett's esophagus and adenocarcinoma. Ann Rev Med 1987; 38: 477-92.

8 Schnell T, Sontag S, Chejfec G, Chintam R, O'Connell S, Kurucar C. High grade dysplasia in Barrett's esophagus. Experience with 36 patients. [Abstract]. Am $\mathcal{F}$ Gastroenterol 1989; 84: 1152.

9 Kerlin P, D'Mellow G, Van Deth A. Barrett's esophagus: clinical, endoscopic and histologic spectrum in fifty patients. Aust NZ F Med 1986; 16: 198-205.

10 Paull A, Trier JS, Dalton MD, Camp RC, Loeb P, Goyal RK. The histologic spectrum of Barrett's oesophagus. $N$ Engl F Med 1976; 295: 476-80.

11 Riddell RH, Goldman H, Ransohoff DF, et al. Dysplasia in inflammatory bowel disease: standardized classification with provisional clinical applications. Hum Pathol 1983; 14: 93168.

12 Reid BJ, Haggitt RC, Rubin CE, et al. Observer variation in the diagnosis of dysplasia in Barrett's esophagus. Hum Pathol 1988; 19: 166-78.

13 Riddell RH. Dysplasia and regression in Barrett's epithelium. In: Spechler SJ, Goyal RK, eds. Barrett's esophagus: pathophysiology, diagnosis and management. New York: Elsevier Science Publishing, 1985: 143-52.

14 Saubier EC, Gouillat C, Samaniego C, Guillaud M, Moulinier B. Adenocarcinoma in columnar-lined Barrett's esophagus. Analysis of 13 esophagectomies. Am $\mathcal{F}$ Surg 1985; 150: 365-9.

15 Schmidt HG, Riddel RH, Walther B, Skinner DB, Riemann JF. Dysplasia in Barrett's esophagus. $f$ Cancer Res Clin Oncol 1985; 110: 145-52.

16 Australian Bureau of Statistics. Hospital morbidity 1988 Brisbane: Australian Government Printer, Catalogue No 4303.3.

17 Brerlow NE, Day NE. Statistical methods in cancer research. Lyon: International Agency for Research in Cancer: 1980 71.

18 Van der Veen AH, Dee J, Blankensteijn JD, van Blankenstein M. Adenocarcinoma in Barrett's oesophagus: an overrated risk. Gut 1989; 30: $14-8$.

19 Achkar E, Carey $W$. The cost of surveillance for adenocarcinoma complicating Barrett's oesophagus. Am $\mathcal{f}$ Gastroenterol 1988; 83: 291-4.

20 Spechler SJ, Robbins AH, Rubbins HB, et al. Adenocarcinoma and Barrett's esophagus. An overrated risk? Gastroenterology 1984; 87: 927-33.

21 Robertson CS, Mayberry JF, Nicholson DA, James PD, Atkinson $M$. Value of endoscopic surveillance in the detection of neoplastic change in Barrett's oesophagus. BrF Surg 1988; 75: 760-3.

22 Hameeteman W, Tytgat GNJ, Houthoff HJ, van den Twee JG. Barrett's esophagus: development of dysplasia and adenocarcinoma. Gastroenterology 1989; 96: 1249-56.

23 Reid BJ, Weinstein WM, Lewin KJ, et al. Endoscopic biopsy can detect high-grade dysplasia or early adenocarcinoma in 
Barrett's esophagus without grossly recognizable neoplastic lesions. Gastroenterology 1988; 94: 81-90.

24 McCallum RW, Jacobson L, Fisher K. Progress report on ACG Barrett's esophagus study. [Abstract]. Am $\mathcal{f}$ Gastroenterol 1988; 83: 1022.

25 Weinstein W, Van Deventer G, Ippoliti A. A histologic evaluation of Barrett's esophagus using a standardized endoscopic biopsy protocol. [Abstract]. Gastroenterology 1984; 86: 1296.

26 Lee RG. Dysplasia in Barrett's esophagus. A clinicopathologic study of six patients. Am $\mathcal{F}$ Surg Pathol 1985; 9: 845-52.
27 Levine DS, Reid BJ, Haggitt RC, Rubin CE, Rabinovitch PS Correlation of ultrastructural aberrations with dysplasia and flow cytometric abnormalities in Barrett's epithelium Gastroenterology 1989; 96: 355-67.

28 Reid BJ, Haggitt RC, Rubin CE, Rabinovitch PS. Barrett's esophagus. Correlation between flow cytometry and histology in detection of patients at risk for adenocarcinoma. Gastroenterology 1987; 93: 1-11.

29 James PD, Atkinson M. Value of DNA image cytometry in the prediction of malignant change in Barrett's oesophagus. $G u$ 1989; 30: 899-905. 